\section{$\underset{\substack{\text { hommes } \\ \text { \& migrations }}}{ }$}

Hommes \& migrations

Revue française de référence sur les dynamiques

migratoires

1286-1287 | 2010

Les migrations subsahariennes

\title{
La migration féminine dans la ville de Kayes au Mali
}

\section{Famagan-Oulé Konaté}

\section{(apenEdition Journals}

\section{Édition électronique}

URL : http://journals.openedition.org/hommesmigrations/1722

DOI : 10.4000/hommesmigrations.1722

ISSN : 2262-3353

\section{Éditeur}

Musée national de l'histoire de l'immigration

\section{Édition imprimée}

Date de publication : 1 juillet 2010

Pagination : 62-73

ISSN : 1142-852X

\section{Référence électronique}

Famagan-Oulé Konaté, "La migration féminine dans la ville de Kayes au Mali », Hommes \& migrations [En ligne], 1286-1287 | 2010, mis en ligne le 01 juillet 2012, consulté le 19 avril 2019. URL : http:// journals.openedition.org/hommesmigrations/1722; DOI : 10.4000/hommesmigrations. 1722 


\section{La migration féminine dans la ville de Kayes au Mali}

Par Famagan-Oulé Konaté, enseignant-chercheur, département de géographie, université de Bamako

Au Mali, la migration n'est plus seulement l'apanage des hommes.

Les femmes maliennes également tentées par l'aventure, seules

ou aux côtés de leur mari, vont à destination d'autres régions du Mali ou vers l'étranger. Malgré la variété de leur profil, des motifs de départ et des activités exercées en zone d'accueil, en prenant la décision de migrer,

elles répondent à une stratégie collective et, à l'instar des hommes, vont à la recherche de ressources matérielles dont une partie est destinée

à leur famille restée au pays. Des données statistiques recueillies dans la ville de Kayes précisent les déterminants de cette migration féminine. 
Au Mali, les données relatives à la migration, appréhendées, d'une part, lors des opérations de recensements généraux de la population et de l'habitat, et, d'autre part, lors de l'Enquête Malienne sur la Migration et l'Urbanisation ${ }^{(1)}$ réalisée dans le cadre du réseau REMUAO ${ }^{(2)}$, sont récentes et permettent d'étudier l'ampleur du phénomène. En plus des migrations internes entraînées par les perturbations écologiques, il existe des migrations saisonnières masculines et féminines vers les centres urbains.

Le Mali est aussi un pays marqué par d'importants mouvements migratoires sur le plan international. "Les pratiques migratoires y sont anciennes car le pays a été le trait d'union entre l'Afrique du Nord et l'Afrique noire. Ces pratiques migratoires se sont amplifiées au cours de la deuxième moitié du $20^{\circ}$ siècle et elles concernent une grande partie de la population malienne ${ }^{(3)}$."

À notre connaissance, la migration féminine, ses déterminants, ses destinations et les transferts effectués n'ont pas été suffisamment abordés. C'est la raison pour laquelle il est urgent de considérer ces aspects à l'échelle locale, notamment, dans la ville de Kayes, chef-lieu de la première région économique ${ }^{(4)}$ du Mali. Il s'agit de préciser les caractéristiques sociodémographiques des émigrées originaires de la ville de Kayes, les motifs de leur départ, les modes de financement de leur voyage et les pays ou aires d'installation auxquelles aboutissent ces trajectoires migratoires. Par ailleurs, les flux monétaires générés par les émigrées et utilisés par les chefs de ménage des familles restées à Kayes invitent à prendre en compte les activités exercées avant et pendant la migration.

L'approche méthodologique s'est appuyée sur la réalisation d'une enquête quantitative, résultat d'une combinaison de méthodes empirique et aléatoire, auprès de chefs de ménage interrogés sur les membres féminins absents de leurs ménages, pour une période de six mois et plus et, qui résident, soit dans une autre région administrative du Mali, soit à l'étranger. En ce qui concerne la partie empirique, nous nous sommes fondés sur l'histoire migratoire féminine recueillie auprès de certains élus communaux de la ville, mais aussi auprès des chefs de quartiers, lors d'entretiens informels, pour collecter leurs opinions sur la migration féminine dans la ville de Kayes, mais aussi pour retenir les quartiers de la ville de Kayes les plus touchés par le phénomène. Il s'est agi des quartiers de Khasso, de Légal Ségou, de Plateau, de Liberté et de Lafiabougou.

Un échantillon de 160 émigrées a été renseigné auprès de 125 chefs de ménage ${ }^{(5)}$. Le traitement informatique de leurs réponses au questionnaire préétabli a permis de mettre au point différents tableaux et graphiques qui ont fait l'objet d'une analyse. 


\section{Le profil des femmes émigrées}

La caractérisation de ces femmes émigrées s'appuie sur trois variables démographiques: l'âge et le niveau d'instruction au départ en migration; la situation matrimoniale avant le départ et actuellement. En ce qui concerne l'âge au départ en migration, nous constatons que le pic des départs $(27,5 \%)$ intervient entre 25-29 ans. Le départ en migration pour les femmes est donc aussi une affaire de jeunes gens. Avant le départ en migration, elles sont nombreuses (52\%) à atteindre le niveau primaire. Nous comptons $22,5 \%$ de femmes ayant atteint un niveau secondaire et plus, $22,4 \%$ d'illettrées, $2,1 \%$ d'alphabétisées et $1 \%$ ayant fait des études coraniques ou de médersa. Pour ce qui est de la situation matrimoniale, l'étude révèle qu'entre le départ en migration et le moment de l'enquête, la proportion de femmes célibataires est passée de $37,5 \%$ à 13,8 \%, celle des femmes en union, de $61,3 \%$ à $81,9 \%$ et celle des veuves de $1,2 \%$ à $2,4 \%$. Par ailleurs, $1,9 \%$ des femmes en union se sont séparées de leur conjoint ou ont divorcé. Le fait que la proportion de femmes mariées soit si forte ne constitue pas une surprise dans le contexte de la nuptialité au Mali : en effet, l'enquête démographique et de santé en 1996 avait déjà souligné que " 84,8 \% des femmes maliennes étaient en union et 1,2\% d'entre elles étaient veuves ${ }^{(7)}$.

\section{Les motifs de départ}

Hormis le fait que la région de Kayes soit considérée à l'échelle nationale comme répulsive, du fait de la péjoration climatique, on peut distinguer deux grandes catégories de causes de la migration féminine dans la ville de Kayes : d'un côté, les motifs sociaux et, de l'autre, les causes économiques. Une grande majorité de femmes (91,2 \%) a émigré pour des raisons sociales. Il s'agit de rejoindre le mari (64,4\% des cas), de poursuivre ses études ailleurs (13,8\%), de rendre visite à un parent $(6,3 \%)$, etc. Ce fait aurait déjà été souligné par Véronique Petit à propos de la migration des femmes du Plateau dogon au Mali: "Pour une raison ou une autre : regroupement familial, mariage, la famille est la première cause des migrations féminines ${ }^{(8)}$ '. À propos des difficultés du regroupement familial, un chef de ménage interrogé sur la question déclare: "Dans certains cas, les durées longues de séparation des maris partis et de leurs femmes restées auprès des parents ( 5 ans et plus) engendrent un lieu de frustrations, qui se fermente au fil du temps avec comme corollaire les divorces et le déchirement du tissu social'. Seulement 8,3 \% des femmes ont émigré pour des raisons économiques comme chercher du travail, faire du commerce ou exercer une activité économique dans la 
restauration, les salons de coiffure, l'art ${ }^{(9)}$, etc. Selon un ancien élu communal de la ville de Kayes,"les opportunités d'emploi, offertes aux jeunes, sont rares". "Même si je n'encourage pas personnellement la migration des jeunes, force est de constater que ni le pays, ni les communes n'ont mis en place des stratégies efficientes de création d'emplois pour retenir les jeunes. De surcroît, nos écoles de formation mettent chaque année près de 100000 diplômés sur le marché de l'emploi, à peine 10000 trouvent un emploi, dans un tel contexte de morosité socioéconomique, il est difficile de retenir nos jeunes."

\section{La décision, les modes de migrer et le financement du voyage}

À la question de savoir "Qui a décidé du départ de la femme émigrée?", 55 \% des chefs de ménage interrogés ont répondu que c'était à la demande du conjoint, contre $23,5 \%$ de candidates qui ont réclamé le départ. Dans 17,5\% des cas, la décision provient de la famille de la candidate ou de celle de son mari. À ce propos, le Rapport mondial sur le développement humain de 2009 souligne que: "les principaux facteurs liés à la structure de l'économie et de la société, qui sont spécifiques à un contexte mais qui évoluent aussi au fil du temps, influent sur la décision de partir ou de rester ${ }^{(10)}$ ". 
Le plus souvent, la femme a été accompagnée de son mari $(58,1 \%)$, d'un membre de sa famille (21,3\%), d'un membre d'une autre famille (3,8 \%), de ses enfants $(1,2 \%)$, et dans 15,6 \% des cas, elle a voyagé seule. D'une part, le chef de ménage a été informé du voyage de la femme émigrée par l'intéressée elle-même (38,8 \%) et, d'autre part, par le chef de la belle famille (38,8\%). Dans certains cas, l'information est venue du mari à l'extérieur $(18,6 \%$ ) et dans d'autre cas, d'une tierce personne (3,8\%).

Le financement du voyage de l'émigrée a été assuré par le conjoint (60\%), la famille du conjoint ou celle de l'émigrée (24,4\%), les enfants de l'émigrée (12\%), la femme elle-même $(10,6 \%$, un autre membre de la famille de la candidate à l'émigration ou de celle du conjoint $(3,8 \%)$.

\section{Une destination de l'émigration féminine multi polarisée}

L'Europe, dont l'Espagne, la France et l'Italie, représente la principale destination des émigrants maliens hors du continent africain. En France, ils ne sont pas très nombreux. On comptait en 2006, 54243 personnes ${ }^{(11)}$ contre 691361 Algériens et 633736 Marocains $^{(12)}$. Certaines migrations inscrites dans la durée sont entraînées par les perturbations écologiques, et d'autres, plus saisonnières, sont motivées par des raisons économiques. Dans les localités d'accueil de l'intérieur comme dans les terres d'accueil de l'extérieur, les migrants s'organisent en associations et en réseaux pour faciliter le financement, l'accueil de nouveaux immigrants et leur insertion économique et résidentielle. Les résultats de notre étude confirment ces aspects, car ces femmes émigrées se destinent soit à d'autres régions administratives du Mali, soit à l'étranger. Parmi les 160 femmes émigrées étudiées, on remarque que 101 ont choisi une destination internationale (Graphique 1).

Pour l'analyse des destinations internationales, nous distinguons 5 aires d'installation: la France, l'Espagne/l'Italie, les États-Unis/Canada/Amérique du Sud, l'Asie et l'Afrique. Cette dernière comprenant l'Afrique de l'Ouest, l'Afrique Centrale, l'Afrique de l'Est, l'Afrique Australe et le Maghreb (Mauritanie, Maroc, Tunisie et la Libye).

En Afrique, c'est l'Afrique Occidentale (22 femmes), qui constitue la première destination des migrantes. Elle est suivie respectivement par ordre d'importance par le Maghreb (13 femmes), et l'Afrique centrale. (8 femmes). Certaines destinations sont, par contre, tout à fait singulières, si l'on peut dire : telle cette ressortissante unique établie en Asie, telle autre, en Afrique de l'Est ou en Afrique Australe. Pour la migration internationale féminine de la ville de Kayes, nous avons eu des 
renseignements sur les pays de transit de 31 femmes sur 101. Le Sénégal, pour des raisons de proximité géographique, vient en tête avec 10 femmes qui y ont transité. Ce pays est suivi par la Côte d'Ivoire avec 9 femmes. Cette situation n'est pas une surprise dans la mesure où ce pays était la principale destination de la migration malienne en Afrique. À ce propos, Philippe Bocquier et Tiéman Diarra indiquaient que : "Linstallation des 735000 personnes de nationalité malienne en Côte d'Ivoire est le résultat d'une longue histoire. Les sociétés malinké et mandé d'origine malienne n'ont pas failli à cette tradition. La recherche de produits forestiers en échange de produits sahéliens fut la raison du déplacement des premiers Mandés vers la Côte d'Ivoire ${ }^{(13)}$." Les autres pays de transit sont la France, l'Espagne, l'Italie, les États-Unis et le Maghreb : la Mauritanie, le Maroc et la Tunisie.

Les destinations des émigrées de la ville de Kayes qui restent au Mali sont également très diversifiées. À l'exception de Tombouctou, cette recherche révèle que toutes les autres régions (Koulikoro, Sikasso, Ségou, Mopti, Gao et Kidal), plus le district de Bamako, constituent des zones d'accueil des migrantes originaires de la ville de Kayes. C'est le district de Bamako qui reçoit le plus grand nombre de migrantes (36 émigrées

Graphique 1 : Destinations internationales (pays ou aire dPinstallation) des femmes émigrées de la ville de Kayes 
sur un total de 59). Ce fait a déjà été révélé par Sékouba Diarra et Pierre Cissé en 2003 : "La population résidant à Bamako en 1998 est ainsi supérieure de 44 fois à l'effectif des résidents qui y sont nés ${ }^{(1)}$." Bamako est suivi par les régions de Sikasso, Ségou et Mopti comme deuxième destination des migrantes internes (18 émigrées sur un total de 59).

\section{Les activités exercées avant et pendant la migration}

Avant de migrer, les femmes, dans leur grande majorité (110 sur 160, soit 68,8 \%), ne se considèrent pas comme des travailleuses. Cependant, elles exécutent des tâches domestiques importantes comme faire la cuisine, s'occuper des enfants, faire la lessive, etc. Il s'agit là de travail non rétribué et c'est pourquoi elles se disent inoccupées. Parmi celles qui se déclarent "occupées", au nombre de 50, on comptait $22 \%$ de salariées, $36 \%$ qui travaillaient dans le secteur de l'artisanat, $36 \%$ dans le secteur du commerce ou de la restauration et 6 \% qui évoluaient dans le domaine de l'art. 
Pendant la migration, l'analyse de la situation d'emploi, toutes destinations confondues, révèle que les femmes émigrées sont au nombre de 89 à s'être déclarées occupées. 17 d'entre elles, soit 19,1 \% de l'échantillon, n'ont pas pu spécifier la nature de leur travail. Parmi celles dont le travail est renseigné ( 72 femmes), elles sont nombreuses à se retrouver dans le secteur du commerce et de la restauration (33,8 \%). Elles sont également 30,3\% à occuper un travail salarié dans les établissements de commerce, dans les aéroports et gares comme celui de balayeuse. Une faible proportion de femmes émigrées, 14,6 \% et 2,2 \%, travaillent respectivement dans les secteurs de l'artisanat et de l'art. Si nous procédons à une analyse de l'emploi selon le lieu de résidence, pour les émigrées internationales, elles sont au nombre de 48 à déclarer exercer un emploi. Parmi elles, 23 sont dans le commerce ou dans la restauration contre 16 dans le travail salarié et 9 dans le secteur de l'art. Pour les migrantes internes du Mali, on constate qu'elles sont au nombre de 24 à exercer une activité salariée.

\section{Les transferts financiers opérés par les émigrées vers la ville de Kayes}

Les envois d'argent constituent la base essentielle de contact avec la famille restée au village. À Kayes, l'argent des migrants est le grenier des familles ${ }^{(15)}$. Nous remarquons que sur les 160 femmes émigrées, 89 d'entre elles (19 au Mali et 70 à l'étranger) ont fait des transferts financiers au profit des familles de Kayes durant l'année 2009 (tableau 1).

Comme attendu, les montants financiers transférés par les migrantes varient selon le lieu de résidence : au Mali ou à l'étranger. Il ressort de l'examen de ce tableau que les montants envoyés à partir de l'étranger sont plus conséquents que ceux de l'intérieur du Mali. En effet, sur les 19 femmes émigrées internes, la majorité d'entre elles (12 sur 19) a fait des transferts financiers de moins de 200000 F CFA au cours de l'année 2009 et aucun transfert n'a atteint 1000000 de F CFA. Dans le même temps, 17 de leurs homologues à l'étranger ont transféré des sommes comprises entre 200000 et 400000 F CFA. Nous constatons également que 14 émigrées, sur un total de 70 à l'étranger, ont procédé à des transferts de 1000000 F CFA et plus à leur famille de Kayes. La migration aurait permis à ces femmes d'accroître leurs revenus et d'en faire profiter les familles restées à Kayes.

Par ailleurs, sur les 70 femmes résidant à l'étranger (37 en France et 23 dans le reste de l'Afrique), nous constatons une situation très contrastée en termes de transferts financiers, car les migrantes installées en France ont envoyé plus d'argent à leur famille de Kayes que leurs homologues d'Afrique (tableau 2). 


\section{Tableau 1. Montant des transferts financiers effectués par les migrantes selon leur lieu de résidence}

\begin{tabular}{|c|c|c|}
\hline Provenance des montants & Effectifs de femmes & Montant envoyé en F CFA \\
\hline \multicolumn{3}{|l|}{ A) MALI } \\
\hline & $\begin{array}{r}12 \\
4 \\
2 \\
1\end{array}$ & $\begin{array}{c}\text { Moins de } 200000 \mathrm{~F} \\
200000 \mathrm{~F} \text { à } 400000 \mathrm{~F} \\
400000 \mathrm{~F} \text { à } 500000 \mathrm{~F} \\
800000 \mathrm{~F} \text { à } 1000000 \mathrm{~F}\end{array}$ \\
\hline Total & 19 & \\
\hline \multicolumn{3}{|l|}{ B) ÉTRANGER } \\
\hline & $\begin{array}{r}18 \\
17 \\
13 \\
5 \\
3 \\
14\end{array}$ & $\begin{array}{l}\text { Moins de } 200000 \mathrm{~F} \\
200000 \mathrm{~F} \text { à } 400000 \mathrm{~F} \\
400000 \mathrm{~F} \text { à } 600000 \mathrm{~F} \\
600000 \mathrm{~F} \text { à } 800000 \mathrm{~F} \\
800000 \mathrm{~F} \text { à } 1000000 \mathrm{~F} \\
1000000 \text { et plus }\end{array}$ \\
\hline Total & 70 & \\
\hline
\end{tabular}

\section{Tableau 2. Montant des transferts financiers effectués par les migrantes depuis l'étranger}

\begin{tabular}{|c|c|c|}
\hline Provenance des montants & Effectifs de femmes & Montant envoyé en F CFA \\
\hline \multicolumn{3}{|l|}{ A) AFRIQUE } \\
\hline & $\begin{array}{r}12 \\
7 \\
0 \\
0 \\
2 \\
2\end{array}$ & $\begin{array}{l}\text { Moins de } 200000 \mathrm{~F} \\
200000 \mathrm{~F} \text { à } 400000 \mathrm{~F} \\
400000 \mathrm{~F} \text { à } 600000 \mathrm{~F} \\
600000 \mathrm{~F} \text { à } 800000 \mathrm{~F} \\
800000 \mathrm{~F} \text { à } 1000000 \mathrm{~F} \\
1000000 \text { et plus }\end{array}$ \\
\hline Total & 23 & \\
\hline \multicolumn{3}{|l|}{ B) FRANCE } \\
\hline & $\begin{array}{r}6 \\
6 \\
12 \\
3 \\
2 \\
8\end{array}$ & $\begin{array}{l}\text { Moins de } 200000 \mathrm{~F} \\
200000 \mathrm{~F} \text { à } 400000 \mathrm{~F} \\
400000 \mathrm{~F} \text { à } 600000 \mathrm{~F} \\
600000 \mathrm{~F} \text { à } 800000 \mathrm{~F} \\
800000 \mathrm{~F} \text { à } 1000000 \mathrm{~F} \\
1000000 \text { et plus }\end{array}$ \\
\hline Total & 37 & \\
\hline
\end{tabular}


Par exemple, pour une remise d'argent d'un montant d'1 million de F CFA à leur famille de Kayes, on compte 8 émigrées en France contre 2 en Afrique. À ce propos, un chef de famille interrogé déclare : "La vie dans le cercle de Kayes, présentement, est difficile à imaginer sans l'apport de la rente migratoire, qui couvre nos besoins dans les domaines de l'alimentation, de la santé et de l'éducation. La plupart des infrastructures existantes résultent de la rente migratoire".

\section{Modes de transferts d'argent et utilisation par les chefs de ménage}

Les modes de transfert utilisés sont nombreux et variés : ils s'appuient sur des réseaux institutionnels comme les banques et les organismes de transfert, mais aussi sur les réseaux sociaux. Les organismes de transfert ont été évoqués dans 53,5\% des envois. Ils sont suivis par ceux effectués lors des visites de l'émigrée à Kayes (39,8\%), puis des moyens qui cumulent le recours aux organismes de transfert et les visites $(4,5 \%$. Les organismes de transfert et le service d'un boutiquier de Kayes (qui livre de la marchandise à la famille de l'émigrée avant de se faire rembourser lors d'un séjour en France) ont été mis à contribution dans une proportion de 1,1\%.

Une grande partie des transferts d'argent $(85,2 \%)$ est utilisée par les chefs de ménage pour l'entretien des familles. Une autre partie $(11,4 \%)$ est destinée à l'alimentation et aux projets de construction à l'intérieur des concessions, tandis que la dernière partie est consacrée à l'achat de bétail ou à la consommation.

\section{Les opinions de la population de Kayes sur la migration féminine}

Les opinions des chefs de ménage sont très contrastées sur le phénomène de migration féminine. En effet, la grande majorité des chefs de ménage interrogés $(91,9 \%)$ est très favorable à la migration féminine contre $8,1 \%$ d'opinions défavorables. Pour les premiers, la migration féminine est positive, car elle permet de conforter l'économie domestique par les différents transferts opérés (48,8\%), aux filles de trouver un mari ailleurs (39,7\%), de poursuivre leurs études dans des spécialités qui n'existent pas au Mali $(5,1 \%)$, d'accéder à de meilleurs soins de santé $(4,1 \%)$ ou d'acquérir des compétences professionnelles (2,3\%).

Pour les seconds, la migration féminine est perçue négativement car elle est l'un des vecteurs de la déstructuration sociale : elle conduirait à la perte du potentiel de 
reproduction de la population de Kayes et accentuerait les disparités économiques entre les ménages avec et sans migrantes. Une dernière raison de cette perception négative des migrations féminines est le déchirement du tissu familial. La mère de l'une des émigrées précise: "Nous, les mères, nous souffrons énormément de l'absence prolongée de nos enfants partis à l'étranger, car l'argent n'est pas un remède à tout. Nous avons aussi besoin de les voir, de les sentir et de les toucher."

\section{Conclusion}

Cette recherche a porté sur l'émigration féminine dans la ville de Kayes. Nous avons tenté de comprendre quelles étaient les caractéristiques sociodémographiques des migrantes et quelle était la motivation de départ en migration. En cours de route, nous avons pu collecter des données sur les trajectoires migratoires, les aires d'installation, les transferts d'argent entre migrantes et zone de départ et les perceptions sur la migration féminine.

Pour ce faire, nous avons pris la concession comme unité de sondage et le ménage, comme unité d'enquête et le chef de ménage a été soumis à un questionnaire. Afin de mieux cerner la problématique de l'émigration féminine, nous avons eu des entretiens informels avec certains chefs de ménage ou de quartier, des élus communaux, qui ont permis de collecter leurs opinions sur le phénomène de migration féminine.

La présente étude confirme le fait suivant, déjà souligné par Sally Findley en 1991 : "Les femmes s'en vont aussi ${ }^{(16)}$." Ce sont surtout des jeunes de 25-29 ans qui sont candidates à l'émigration. Dans notre échantillon, elles sont majoritairement en union (61,3\%). Par conséquent, la famille, à travers le regroupement familial, est la première cause des migrations féminines dans la ville de Kayes.

Il est nécessaire de rappeler que la décision de migrer est avant tout une affaire du groupe, avec le mari comme acteur principal. Cette situation n'exclut pas pour autant des choix personnels de migrer pour réaliser des projets individuels. Notre échantillon révèle aussi que la France constitue la principale destination des migrantes internationales de la ville de Kayes, en dehors du continent africain. Mais il semblerait que les pays d'accueil, à l'image de la migration internationale masculine, se diversifient géographiquement ${ }^{(17)}$.

Le départ d'un membre du ménage n'est pas forcément synonyme de rupture sociale. En effet, les absents entretiennent avec la localité de départ d'importants contacts variés : envois d'argent, de biens en nature, visites, appels téléphoniques etc. Les envois d'argent constituent la base essentielle du contact avec la famille restée au 
village. Ils sont destinés prioritairement à l'entretien des ménages ou des familles restés sur place : l'alimentation, la santé et l'éducation des enfants.

Les opinions de la population divergent sur la migration féminine. Certains chefs de ménage estiment qu'elle n'a que des avantages. Leur argumentation se fonde sur les transferts matériels et les remises d'argents opérés par les migrantes pour leur famille restée à Kayes. D'autres, au contraire, nous pouvons le dire, sont farouchement opposés à cette migration féminine, en arguant qu'elle conduirait à la perte du potentiel de reproduction de la population de Kayes, et accentuerait les disparités économiques entre les ménages qui comptent ou non des migrantes.

\section{Notes}

1. EMMU, Enquête malienne sur la migration et l'urbanisation en Afrique de l'Ouest. Rapport descriptif national, Bamako, CERPOD/DNSI, 1996.

2. REMUAO : Réseau Migration et Urbanisation en Afrique de l'Ouest.

3. Traoré Sadio, "Les nouvelles tendances migratoires en Afrique de l'Ouest", in Véronique et Seydou Keita (coord.), Questions de population au Mali, Bamako, Le figuier, 2003.

4. Le Mali est divisé en huit régions économiques : Kayes, Koulikoro, Sikasso, Ségou, Mopti, Tombouctou, Gao, Kidal, plus le district de Bamako.

5. Groupe d'individus qui vivent ensemble et mettent en commun tout ou partie de leurs ressources pour subvenir à leurs besoins essentiels ("Niakhamé" en soninké).

6. Dans la présente étude, toutes les femmes en union ont leur mari en dehors de Kayes, soit dans une autre région du Mali, soit à l'étranger.

7. Coulibaly Salif, Dicko Fatoumata, Traoré Seydou Moussa, Sidibé Ousmane, Seroussi Michka et Barriere Bernard, Enquête Démographique et de Santé Mali, Calverton, Maryland USA, DNSI/ Macro International Inc., 1996.

8. Petit Véronique, Migrations et société dogon, Paris, L'Harmattan, 1998.

9. Au Mali, beaucoup de femmes se déclarent inoccupées, car le travail domestique dont elles ont en charge comme faire la cuisine, entretenir les enfants, faire la lessive n'est pas rémunéré.

10. Nations unies, 2009.

11. Évidemment, ce chiffre ne reflète pas toute la réalité, car une autre partie des migrants relève de la migration clandestine.

12. Insee, Recensement de la population, janvier 2006

13. Bocquier Philippe et Diarra Tiéman, Population et société au Mali, Paris, L'Harmattan, 1998.

14. Diarra Sékouba et Cissé Pierre, "Migrations et pauvreté au Mali”, in Véronique et Seydou Keita (coord.), Questions de population au Mali, Bamako, Le figuier, 2003.

15. Gubert Flore, "La participation des Maliens de France au développement de la région de Kayes", in Bocquier P. et al, op. cit, pp. 103-121.

16. Findley Sally, "Les femmes s'en vont aussi", in Revue Pop-Sahel, ${ }^{\circ} 4$, Bamako, CERPOD/INSAH/CILLS, 1991.

17. Avec la nouvelle donne restrictive de la politique d'immigration de l'Union européenne, la migration internationale de la région de Kayes est en train de s'ajuster et de se diversifier (même si l'Europe va continuer à demeurer, pour un certain temps, la principale destination). 